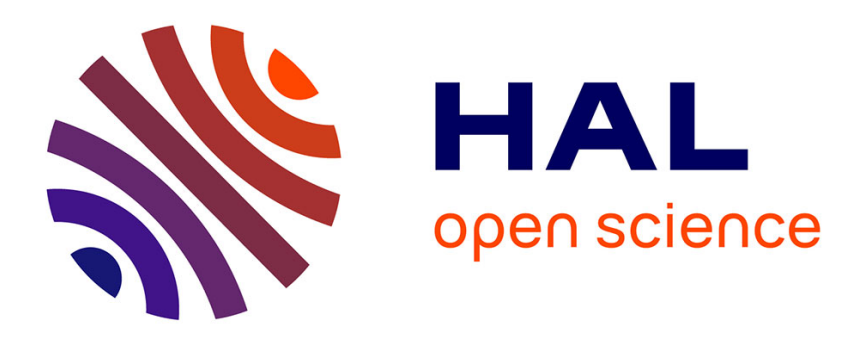

\title{
Application of the curvelet transform for pipe detection in GPR images
}

Guillaume Terrasse, J. M. Nicolas, Emmanuel Trouvé, Émeline Drouet

\section{To cite this version:}

Guillaume Terrasse, J. M. Nicolas, Emmanuel Trouvé, Émeline Drouet. Application of the curvelet transform for pipe detection in GPR images. IGARSS, Jul 2015, Milano, Italy. hal-01187681

\section{HAL Id: hal-01187681 \\ https://hal-imt.archives-ouvertes.fr/hal-01187681}

Submitted on 7 Sep 2015

HAL is a multi-disciplinary open access archive for the deposit and dissemination of scientific research documents, whether they are published or not. The documents may come from teaching and research institutions in France or abroad, or from public or private research centers.
L'archive ouverte pluridisciplinaire HAL, est destinée au dépôt et à la diffusion de documents scientifiques de niveau recherche, publiés ou non, émanant des établissements d'enseignement et de recherche français ou étrangers, des laboratoires publics ou privés. 


\title{
APPLICATION OF THE CURVELET TRANSFORM FOR PIPE DETECTION IN GPR IMAGES
}

\author{
Guillaume Terrasse ${ }^{1}$, Jean-Marie Nicolas ${ }^{1}$,Emmanuel Trouvé ${ }^{2}$, Émeline Drouet ${ }^{3}$ \\ guillaume.terrasse@telecom-paristech.fr \\ ${ }^{1}$ Institut Mines-Télécom, Télécom ParisTech CNRS-LTCI, Paris, France \\ ${ }^{2}$ Université Savoie Mont Blanc, Polytech Annecy Chambéry, LISTIC, Annecy, France \\ ${ }^{3}$ ENGIE, Direction Recherche et Innovation, Saint-Denis La Plaine, France
}

\begin{abstract}
This paper is dedicated to the detection of buried pipes with a ground penetrating radar (GPR). The images from GPR acquisitions also called B-scan are corrupted by clutter and noise. In order to remove these undesirable items we propose to use the properties of the curvelet transform. We're using this method as a first step of the automatic detection of hyperbola in a B-scan.
\end{abstract}

Keywords : GPR, clutter removal, denoising, curvelet transform.

\section{INTRODUCTION}

In order to detect the pipes, four technologies are currently used for the detection of buried utilities. These four tools are the low-frequency electromagnetic, the RFID technology, a method based on the use of acoustic waves and the ground penetrating radar (GPR). In this work we are interested in the improvement of GPR images.

The ground penetrating radar has been widely used in different applications like civil engineering, geological study or glaciology ([1]). This devise sends electromagnetic pulses with a large band and a high frequency, usually between $100 \mathrm{MHz}$ and $2 \mathrm{GHz}$. Then, the receiving antenna records the backscattered wave at each position as function of time. This function is called an A-scan. Finally, all the A-scans recorded at different positions form an image called B-scan in which each pixel intensity corresponds to the amplitude of the backscattered wave at a certain position of recording and time.

Important amount of information is received from all the objects which reflect the radar wave. In order to detect and localize the pipes, we want to suppress the clutter, namely all useless echoes which could hide those from pipes. In this paper, we present a method to remove these noise and the clutter. We present experimental results obtained on simulated data and on real GPR images.

This work was supported by the "G4M" project which provided a GPR and access to ENGIE-CRIGEN test area

\section{GROUND PENETRATING RADAR}

Thanks to its ability to send an electromagnetic pulse in ground, the GPR provides information depending on the distribution, nature and composition of the materials of subsurface. Echoes are created when the wave encounters an heterogeneity in soil. The shape of the ensemble of echoes on a B-scan and their intensity can give us an indication of the nature of the object. On one hand, the presence of an hyperbolic shape means there is a punctual-like object, for instance a pipe or a rock. On the other hand, a linear shape could be a boundary between two layers. Due to its large band, the GPR allows the subground to be mapped with accuracy, but it also collects a lot of noise that has to be reduced to make the data more understandable. There are several works about denoising of GPR data. Some of them have used the wavelet transform and a threshold on coefficients, a frequency filter (elliptic, Butterworth filter) [1], [2] or a time domain filter (mean/median filter) [1].

The B-scan is composed of all elements which reflect the GPR pulses. Most of the clutters come from the direct wave, the reflection on a different layers and its multi-echoes. They can be recognized by their strong amplitude and/or their horizontal structure. Based on this observation, several studies try to exploit the high correlation between A-scans to suppress these types of clutter. For example, the subtraction of a mean A-scan, processing in the $\mathrm{f}-\mathrm{k}$ domain with the use of a declivity filter [3] or an high-pass filter [4], a singular value decomposition [5] or the use of the curvelet transform [6].

\section{METHOD PROPOSED}

In the case of the direct wave which have a perfect horizontal shape, the method proposed in [4] shows that we can remove it with an high-pass filter. Indeed the frequency content of the hyperbola is higher than the direct wave and so we can filter it with a suitable choice of the cutoff frequency.

To have more freedom about the element to remove and not only the zero slope structure, our method intend to take a slope parameter into account, like the declivity filter. For these reasons, we used an oriented wavelet transform which provides both a frequency and an angular decomposition. In addition to the clutter, noise is present in the data. It's also important to remove it in a pre-processing step to ensure the good quality for the following analysis. 
Moreover, we need a computationally efficient processing to be used in real-time. Thus we choose to use the curvelet transform and particularly we apply the Fast Discrete Curvelet Transform (FDCT) algorithm [7].

This transform was used in several domains, for the classification [8], the denoising [9] [6] or the clutter removal [6] of images and seismic or GPR data .

\subsection{Why the curvelet transform?}

Curvelet is a particular 2D wavelet with a high anisotropic property. It's a multi-scale analysis tool and allows a timefrequency and directional localisation. It offers an optimal sparse representation of a wave propagator [7]. So it seems theorically adpated to GPR data. It also provides a sparser representation of an image and a better display of the edges than a classic wavelet transform. Figure 1 (a) presents the difference between wavelet (left) and curvelet transform (right) for the representation of a curve and shows how curvelet transform provides a sparser representation of a curve than wavelet transform.
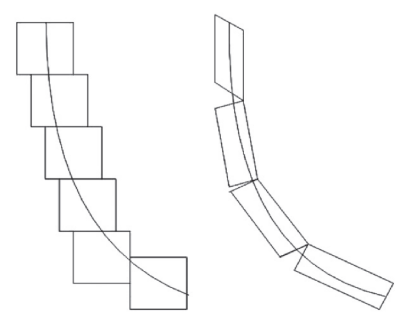

(a) Curve representation by wavelet (left) and curvelet (right) [6]

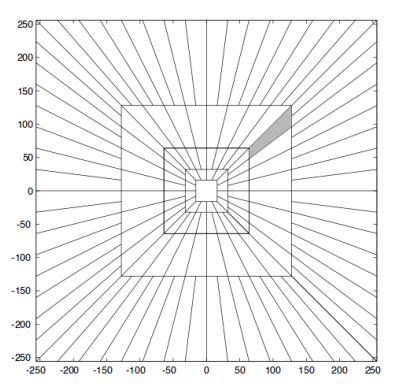

(b) Fourier space tiling [7]
Fig. 1: Curvelet transform properties

The FDCT is divided into several steps. Firstly, we take the Fourier transform of the image, then we divide it into tiles of concentric squares (Fig 1(b)). Each tile is translated to the origin and wrapped with a rectangle of same support. Finally we compute its inverse Fourier transform and get it as curvelet coefficients. Therefore after the computation of the FDCT, we obtained a collection of curvelet coefficients spread according to the support and the position of their tile.

\subsection{The clutter removal processing}

We intend to reduce a particular clutter which has an horizontal shape whilst keeping the point-like reflector. For that, we use the curvelet transform and we exclude the horizontal curvelets which are employed for the representation of the clutter.

Firstly, we have studied the energetic distribution of the angular panels in each level of detail. It corresponds to the distance between the tile of interest and the origin in the Fourier domain; closer the tile of origin, coarser is the level of detail.
We have applied the FDCT to a simulated B-scan with only an hyperbola and a second one with an additional ideal clutter (cf Figure 2).

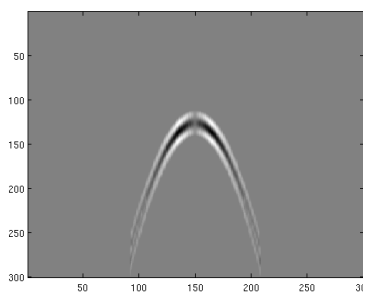

(a) Hyperbola

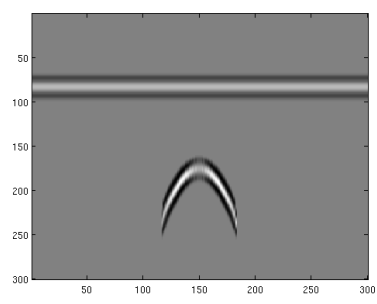

(b) Hyperbola + clutter
Fig. 2: Simulated B-scan

Figure 3 presents the energetic distribution of the curvelet coefficients at the second detail layer for the two toy examples showed in Figure 2. The angular panels 8, 9, 40 and 41 present a high energetic value for the toy example with a clutter, whereas this energy is very weak for these panels in the example without clutter. So we conclude that the clutter and hyperbola informations can be well separated in the curvelet space.

The clutter has an important low frequency information and therefore it's widely defined in the coarsest layer. Thus, by substituting the value of these coefficients by zero we should strongly reduce the clutter. However it also removes a part of the useful signal, mainly at the upper part of the hyperbola. We also substitute all the coefficients corresponding to a zero slope curvelet by zero.

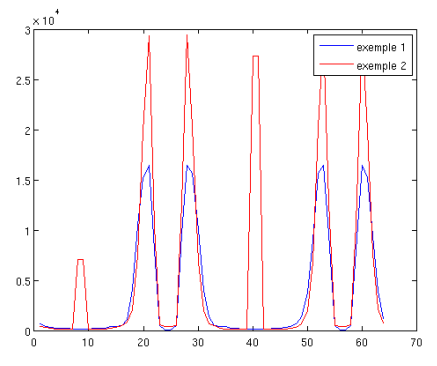

Fig. 3: Energetic distribution in function of the angular panel, blue : without clutter; red : with clutter

\subsection{Denoising}

Most of the studies about denoising of B-scan use a filter processing or the wavelet transform. In [6], the curvelet transform is employed with a threshold of the coefficients in order to denoise the data. The inconvenient of this method is the need to estimate the variance of the noise.

As explained above, the curvelet transform decomposes the B-scan in several levels. We noticed that the finest layer, with the high frequency information, contains the majority of the noise and no or almost no useful information. 
Sometimes we also observe some annoying elements like columns (Figure 7 (a)). Such elements can be removed by using the directional localization properties of the curvelet transform. As for the clutter removal method, an efficient way to remove the noise is to eliminate all the coefficients in each layer corresponding to a vertical curve and in the finest level.

\section{RESULTS}

17 B-scan images have been acquired by an USRADAR GPR with an $500 \mathrm{MHz}$ antenna on a test area where the nature of soil and the position of pipes are known.

Our results are presented from B-scans where two examples are shown in Figure 4. In Figure 4 (a) we show a GPR image, where there are five pipes. The first three from left to right are made of polyethylene (PE) and the following two of cast iron and steel. The pipes have a diameter of $16 \mathrm{~cm}, 2 \mathrm{~cm}$, $6.3 \mathrm{~cm}, 11.8 \mathrm{~cm}$ et $16 \mathrm{~cm}$ respectively from left to right. Three of them are quite easy to see but the two others are more difficult to distinguish. The second B-scan (Fig 4 (b)) shows two pipes made of PE (on the left) and of steel (on the right) with a diameter of $11 \mathrm{~cm}$ and $2 \mathrm{~cm}$ respectively.

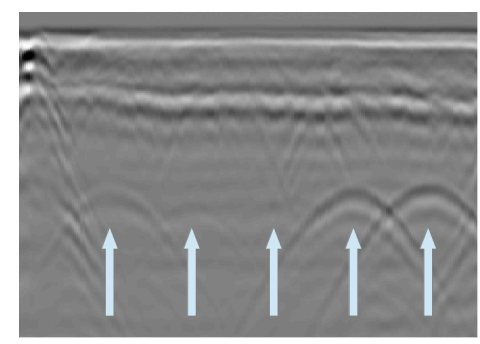

(a) B-scan with 5 pipes

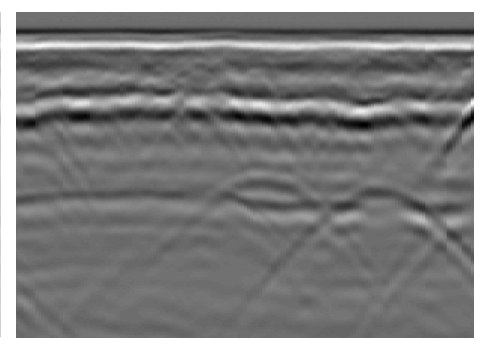

(b) B-scan with 2 pipes
Fig. 4: Two examples of original B-scans

\subsection{Clutter removal}

Firstly we have applied the proposed clutter removal method. The result is presented in Figure 6 (a) and (b). In Figure 5, we present a comparison of the signal to clutter ratio (SCR) for $17 \mathrm{~B}$-scan images for different methods, the mean subtraction (MS), window mean subtraction (WMS), singular value decomposition (SVD), declivity filter (DF), the highpass filter (HP) and our method (CRVT) .

To assess performances, the energy of the clutter and of the hyperbolas after processing $\left(I_{p r o c}\right)$ is computed and normalised by the original energy $\left(I_{\text {org }}\right)(\mathrm{Eq} 2)$. Then, SCR is computed from the normalised energy of the hyperbolas divided by the normalised energy of the clutter. The energy of the different items are measured from patterns which fit them. $\mathrm{X}$ and $\mathrm{Y}$ define the area of the pattern.

$$
\begin{aligned}
E & =\sum_{x \in X} \sum_{y \in Y(X)} \frac{I_{\text {proc }}(y, x)^{2}}{I_{\text {org }}(y, x)^{2}} \\
S C R & =\frac{E_{\text {hyperbolas }}}{E_{\text {clutter }}}
\end{aligned}
$$

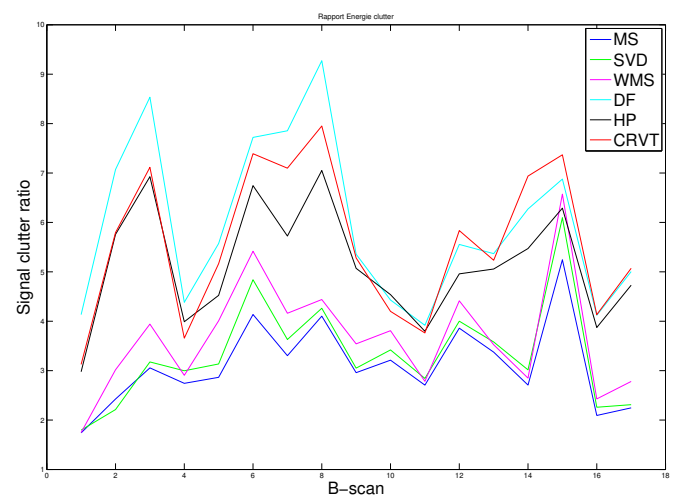

Fig. 5: Signal to clutter ratio for the $17 \mathrm{~B}$-scans

In Figure 5, one can observe, that in most of the cases the declivity filter obtains a better signal to clutter ratio than the other methods. But overall the declivity filter and the method proposed are close. This is explained by the fact that both methods are conceptually close. Indeed, they filter the frequency space with a cone centred at the origin and its principal axis coincides with the y-axis. Nevertheless our method has reduced the number of input parameters in comparison of the declivity filter. The declivity filter needs at least three parameters whereas our method only needs one which is easy to interpret because it corresponds to the slope that we want to remove.

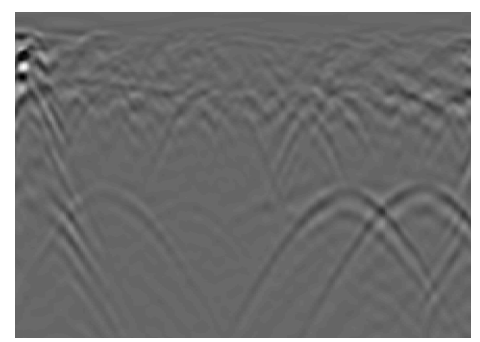

(a) B-scan with 5 pipes

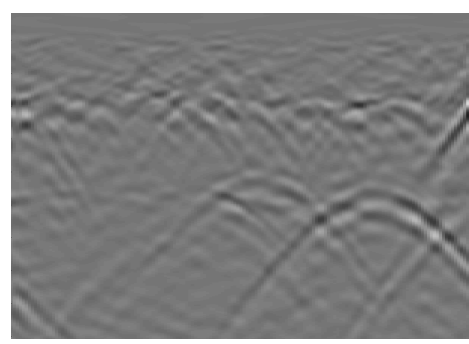

(b) B-scan with 2 pipes
Fig. 6: Result of the clutter removal

We also notice in Fig 6 (a) and (b) that the zero-slope elements in the background have been removed. The B-scan of Fig 6 (b) shows a layer boundary which has been removed without having a significant impact on the hyperbolas.

\subsection{Denoising}

In this subsection, we present the result for the denoising method. Figure 7 (a) shows the enlarged area from a B-scan acquired with a GSSI GPR which contains noise and column effects. In Figure 7 (b) to (e) we compare our processing with different denoising methods for the noise and column effects removal. At first with 1D methods, we process each column of the B-scan from a discrete wavelet transform (DWT) with a Daubechies 6 and a butterworth filter in frequency space. Then with 2D methods, from a mean sliding window and the thresholding of the curvelet coefficients and lastly the proposed method. 


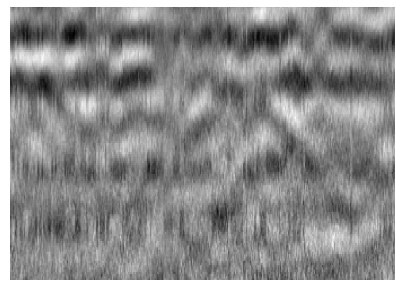

(a) Noisy B-scan

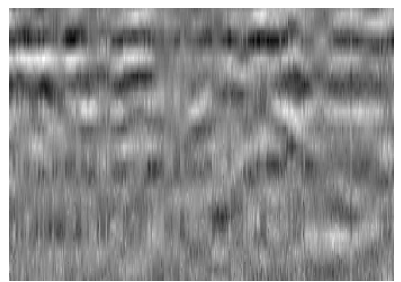

(b) DWT

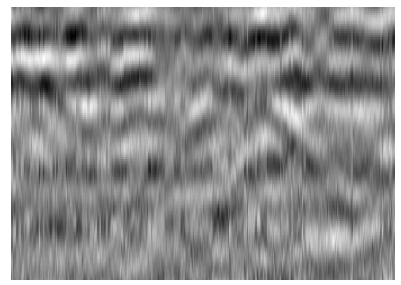

(c) Freqential filter

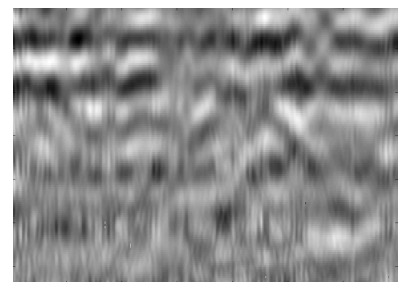

(d) Curvelet threshold

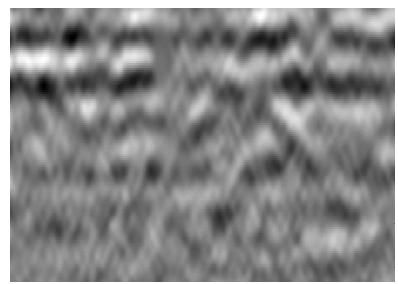

(e) Our Method

Fig. 7: Qualitative results of denoising and column effects removal

In order to assess the performances of the denoising (without column) of our method, we compute for each processing the signal to noise ratio (SNR)(Eq. 3), the peak signal to noise ratio $(\mathrm{PSNR})(\mathrm{Eq}$. 4) and the normalised root mean square error (NRMSE)(Eq. 5). Higher SNR and PSNR values imply better results. On the contrary, a method will be better when the NRMSE is close to zero. For this, we use a part of a B-scan where the noise is negligible which will be our reference (x) and we add on it a centred white gaussian noise (y). The results of the denoising are presented in Table 1.

$$
\begin{gathered}
S N R=20 \log _{10}\left(\frac{\sum_{i=1}^{N} x[i]^{2}}{\sum_{i=1}^{N}(x[i]-y[i])^{2}}\right) \\
P S N R=10 \log _{10}\left(N \frac{\max (\mathbf{x}, \mathbf{y})}{\sum_{i=1}^{N}(x[i]-y[i])^{2}}\right) \\
N R M S E=\sqrt{\frac{\sum_{i=1}^{N}(x[i]-y[i])^{2}}{\sum_{i=1}^{N}(x[i]-\bar{x})^{2}}}
\end{gathered}
$$

\begin{tabular}{|l||c|c|c|}
\hline & SNR & PSNR & NRMSE \\
\hline DWT & 24 & 31 & 0.27 \\
\hline Butterworth & 25 & 31 & 0.24 \\
\hline Mean Filter & 30 & 27 & 0.13 \\
\hline T curvelet & 33 & 40 & 0.09 \\
\hline Our method & $\mathbf{3 3}$ & $\mathbf{3 9}$ & $\mathbf{0 . 1}$ \\
\hline
\end{tabular}

Table 1: Denoising Results

In Figure 7, we present the qualitative results of denoising and the column effects removal. It can be easily noticed that the result obtained by our method is better than the other methods without any parameter to choose or the variance of the noise to estimate. In Table 1, the two curvelet methods get the best results for denoising without column effects.

\section{CONCLUSION}

In this paper, we presented a method to remove the clutter and the noise of GPR images using curvelet transform. It can be computed for the clutter removal or the denoising together or separately. It's fast (two seconds for a B-scan of $512 \times 2000$ ) and the only parameter we need, is an a priori on the maximal slope of the clutter, due to the slight oscillations.
Moreover the proposed method allows a better highlighting of the signals with a low energy to suppress a particular noise with a column effects.

Nevertheless, this processing remove a part of the useful signal, mainly at the top of the hyperbola. Therefore, the future work will focus on the coefficients in each angular panel to avoid to reduce the energy on the top of the hyperbola. This method will be used in a pre-processing step for the automatic detection of the hyperbolas.

\section{REFERENCES}

[1] H.M. Jol, Ground penetrating radar, theory and applications, Elsevier, 2009.

[2] J. Baili, S. Lahouar, M. Hergli, I.L. Al-Qadi, and K. Besbes, "Gpr signal de-noising by discrete wavelet transform," NDT \& E International, vol. 42, no. 8, pp. 696 - 703, 2009.

[3] S. Perrin, Contribution à l'Algorithmique Multicapteur pour la Détection de Mines Antipersonnel, Ph.D. thesis, École Centrale de Lille et Université des Sciences et Technologies de Lille, 2001.

[4] D. Potin, E. Duflos, and P. Vanheeghe, "Landmines groundpenetrating radar signal enhancement by digital filtering," IEEE Geoscience and Remote Sensing Society, pp. 2393 - 2406, 2006.

[5] P.K. Verma, A.N. Gaikwad, D. Singh, and M.J. Nigam, "Analysis of clutter reduction techniques for through wall imaging in uwb range," Progress In Electromagnetics Research B, vol. 17, pp. $29-48,2009$.

[6] Z. y. Zhang, X. d. Zhang, H. y. Yu, and X. h. Pan, "Noise suppression based on a fast discrete curvelet transform," Journal of Geophysics and Engineering, vol. 7, pp. 105-112, Mar. 2010.

[7] E. Candès, L. Demanet, D. Donoho, and L. Ying, "Fast discrete curvelet transforms," 2005.

[8] J. Luo, D. Song, C. Xiu, S. Geng, and T. Dong, "Fingerprint classification combining curvelet transform and gray-level cooccurrence matrix," Mathematical Problems in Engineering, p. 15, 2014.

[9] J.-L. Starck, E.J. Candès, and D.L. Donoho, "The curvelet transform for image denoising," IEEE Transactions on Image Processing, vol. 11, no. 6, pp. 670-684, 2002. 